\title{
Modeling of Carbonated Metabolites Production by Clostridium butyricum W5 Under Anaerobic Conditions for Optimization of Hydrogen Metabolic Production
}

\author{
Andres C. Diaz Valencia ${ }^{1, *}$, Gustavo A. Garzon Fajardo", Fabio E. Sierra Vargas ${ }^{2}$ and \\ Carlos A. Guerrero Fajardo ${ }^{3}$
}

${ }^{1}$ Universidad Nacional de Colombia, Sede Bogotá, Facultad de Ciencias, Departamento de Biologia, Carrera 45 No. 26 - 85 Bogotá, Colombia

${ }^{2}$ Universidad Nacional de Colombia, Sede Bogotá, Facultad de Ingenieria, Departamento de Ingenieria Mecanica, Colombia

${ }^{3}$ Universidad Nacional de Colombia, Sede Bogotá, Facultad de Ciencias, Departamento de Quimica, Colombia

\begin{abstract}
Hydrogen production has been one of the most studied problematics nowadays because of the worldwide needs of change in energy sources for daily use, industrial use and laboratory use. In addition, we can also mention the importance of this energy sources to be clean, self-sustainable and of course profitable for the industry, that is the reason why in this work we studied one of the most common hydrogen-producer microorganisms, Clostridium butyricum. This bacterial specie was chosen because it's metabolic pathways have been widely studied and it's an easy-obtainable microorganism present in almost any kind of anaerobic environment such as some zones of soil, human intestines and others, in addition to that, we can also mention some other species for hydrogen production: Kleibsella pneumoniae and Citrobacter freundii which can also be used for the modelation process and the industrial process of production. In this work we developed a process model about the metabolic pathways of $C$. butyricum in order to understand the hydrogen production process and reveal the points where it can be optimized. Mainly, the model was designed so it could predict the comportment of the process in the exponential phase of growth of the bacteria. Stoichiometric analysis was performed in order to create theoretical curves of the production of hydrogen and other metabolites. Part of the study was performed based in experimental results exposed by other authors, the model was developed in JMCAD ( an opensource software for complex dynamic system modeling based in Java. The predicted results are comparable with the experimental ones as it can be seen in the results section, the $R^{2}$ of all the curves was (near) to 0,97 so we could ensure a close relation with the reality. Some suggestions in order to maintain the exponential phase of growth in the cultures of C. butyricum are exposed and explained further in the article.
\end{abstract}

Keywords: Hydrogen production, C. butyricum, Mathematical modeling, JMCAD, Quorum sensing, Stoichiometric analysis.

\section{INTRODUCTION}

Worldwide energy sources until today have been fossil-related fuels and chemical-synthesized fuels, energy sources that have been useful and quite effective. The issues come when we start to consider the damages that have been made in the planet and in addition when we consider that that kind of energies are nonrecoverable and so they are not selfsustainable, that is why nowadays the world is in need of acquiring a new energy source, one that in addition to be self-sustainable is friendly with the environment and even more, one that is easily obtainable and that in an ideal situation can help out with the damages of our planet.

Hydrogen is one kind of energy source that actually full-fills the above mentioned requirements of the

*Address corresponding to this author at the Universidad Nacional de Colombia, Sede Bogotá, Facultad de Ciencias, Departamento de Biologia, Carrera 45 No. 26 - 85 Bogotá, Colombia; Tel: +57(1)3165000;

Fax: +57(1) 3165000 ext. 10987; E-mail: acdiazv@unal.edu.co "ideal" energy source except for two of them: its production by physico-chemical intensive and nonclean ways, and its high production costs. For these reasons it is necessary to research in alternatives ways for hydrogen production, such as biological, using microorganisms that produce hydrogen as a normal metabolite.

C. butyricum produces hydrogen in an anabolic metabolic pathway [1], the anaerobic acid-mixed fermentation of glucose. Principal metabolites generated are: butyrate, acetate, carbon dioxide and diatomic hydrogen, in addition to other metabolites which presence or absence depends of the strain used in the culture, those metabolites are: lactate, formate and ethanol between others.

As this bacterial species use glucose for the production of hydrogen [2] we can infer that almost any glucose source must theoretically act as an efficient substrate for culturing. his is a promising fact due to the 
big pantone of possibilities that this opens to us, one of them, and the ideal one is actually located in countries where the economy depends on agriculture because in that kind of economic activities the waste of organic material is really considerable and in addition to that the most sophisticated use of it is as compost or manure.

The previous statement implies that if a biological hydrogen production process is standardized, analyzed, optimized and proposed in those countries, the use of organic material is going to represent an economic income for them and their people, in addition hydrogen production would supply some of society needs.

Hydrogen is considered an energy clean source and has a lot of additional uses such as:

1. Reactant in laboratory synthesis processes in order to crack hydrocarbons its or generate hydrogenation of compounds. [3]

2. Coolant in electronic mechanisms.

3. Generation of water vapor when combusted in order to be collected or be implemented in other uses.

After this considerations we can actually see that it is really important to get in the deep investigation of the natural production sources such as cyanobacteria and algae.

\subsection{Modeling and its Implications in the} Development of the Industry

Before the performance of any experimental activity is strongly suggested to have a theoretical approach to the activity, not only to work at laboratory scale, but an industrial scaling too, so that, the production of hydrogen with fermentative bacteria could be optimized easily. An analysis of the growth kinetics, rates an general behavior joined to a biochemical stoichiometric analysis of the metabolic pathway of fermentation might provide a well based model in which the process could be altered and so optimized in order to acquire the most out of it.

\section{MATERIALS AND METHODS}

\subsection{Selection of Growth Curves and Kinetics}

From previous published works in $C$. butirycum growth kinetics, the growth curve was selected following a set of basic requirements that are listed below:

- It must be the result of an experimental process so the data acquired from it was as close to the reality as possible.

- It must to be described in CFU's versus time or $O D_{600}$ versus time.

If it is possible, time must be expressed in minutes instead of hours

If it is possible it must contain the growth rates in order to be compared to the theoretical ones generated by the model.

Once the curve was selected it was standardized and data was obtained in order to find a linear, exponential, logarithmic or power regression that described its behavior.

\subsection{Formation of Curves}

The curves were generated and analyzed in LibreOffice Calc (part of The Document Foundation 3.3.4 O00330 m19 (Build:401) tag libreoffice-3.3.3.1, Ubuntu package 1:3.3.4-Oubuntu1.4) the $R^{2}$ values were calculated in the same software and checked in ARC v. 1.6 in order to confirm the accuracy of the values obtained.

\subsection{Stoichiometric Analysis}

The stoichiometric analysis was performed according to the normal procedures and based in the metabolic pathway (Figure 2) of anaerobic fermentation of glucose in Clostridium butyricum that is shared by strains like $C$. butyricum W5 and $C$. butyricum WT.

The stoichiometric proportions of the analysis were calculated and verified with stoichiometric proportions reported by other authors, $100 \%$ efficiency was used for the analysis in order to ensure that the modeled process was completely modifiable without altering the proportions.

\subsection{Determination of Hydrogen Production Curves, Equations and Stoichiometric Proportions}

Hydrogen production was determined by verifying the stoichiometric proportions found with the ones reported experimentally by other authors, the experimental ones were chosen due to the hight proximity with reality processes. 


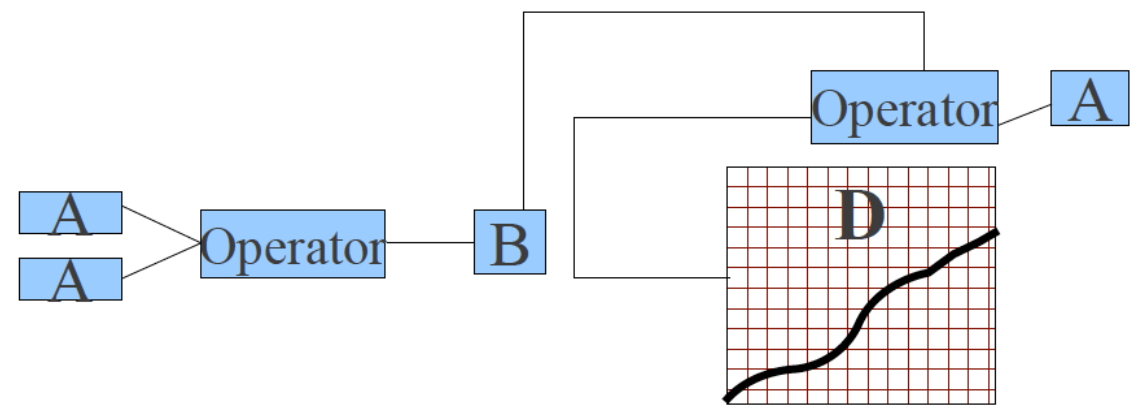

Figure 1: Model design interface (model shown is for illustrative purposes only). Interface shows programming tools such as operators, variables, constants and reporters (graphic and numeric outputs). A: Variables, constants. B: Numeric Reporter, D: Graphic reporter.

Hydrogen production curve was generated with the data acquired about rates, times of production and cell density, in the same way as the growth curve it as standardized and used to create a linear, exponential, logarithmic or power regression that best described its behavior.

The production of the rest of the metabolites of interest (butyrate, acetate and carbon dioxide) was calculated in base of the tendency line calculated for the curve.

\subsection{Discrimination of Curves}

In order to ensure the maximum proximity to reality all the regression curves (or lines) calculated were discriminated according to the $R^{2}$ values obtained, all curves with $R^{2}$ values under 0.987 were considered of bad quality as they don't reach the requirements of reality approximation.

\subsection{Values Reaching of Asymptote Prevention}

As the regression curves calculated tend to have one or more asymptotes the reaching of those points must be prevented, in order to accomplish this we limited the time development of the model so the results obtained were totally reproducible exclusively in the exponential phase of growth although equations can still be used in models in which the exponential growth of the cells is maintained and might be useful when trying to predict the final cell concentration depending of a first inoculation.

\subsection{Model Development}

The model to describe the behavior of all the mentioned variables according to the time was completely programmed in JMCAD, an open-source software for complex dynamic system modeling, based on Java, in order to accomplish the objectives of it the equations obtained for each one of the variables were related one with another so they could be synchronized and could present the desired results.

Each one of the factors in the equations were considered constants so the standard behavior of the system couldn't be modifiable, the main variable considered was the time in order to see in simulated time the complete development of the processes of interest such as production and accumulation of hydrogen, acetate, butyrate and carbon dioxide, in addition to that the values of substrate and stoichiometric proportions are also modifiable in order to understand and analyze the critic points of the model and therefore the critic points of the production process. Simulations were ran in JMCADRTS and JMCADRTC (JMCAD Real Time Schema and JMCAD Real Time Control) (See Figure 1).

\subsection{Data Storage}

The data from the simulation was stored at time steps (each 70 simulated minutes) in order to acquire data points that could be useful to verify the equations used in the model and thus confirm the match or mismatch of the results.

The above mentioned information was stored for the creation of a static curve that could be compared with the static curve of the theoretical non-simulated results.

\subsection{Relations Diagram}

The relations diagram of the production system was designed in Vensim PLE v5.11A.

The relations diagram illustrates the relations within cell production and all the metabolites produced in function of this, all of this relations are shown in Figure 4. 


\subsection{Modeling Paradigm}

The modeling software is based in programmation oriented to objects, in this software equations were designed and established as blocks, blocks are pieces of the software that interact as independent systems with inputs and outputs, each variable and operator of the system was connected with the others in order to configure the complex non-linear system.

In addition to that software was debugged by running several simulations with JMCADRTS (JMCAD Real time schema) and checking the results with the result of calculations coming from the equations designed.

\subsection{Special Units Definition and Variable Names Definition}

$\mathbf{R}^{2}: \quad$ Correlation index (Statistics)

CFU's: Colony forming units count. (cell density unit)

Mmol: $\quad$ Mili-moles $\left(\mathrm{mol} \cdot 10^{-3}\right)$

T: $\quad$ Temperature.

P: Bacterial concentration.

N: Initial inoculation.

Tc: Growth rate.

V: $\quad$ Hydrogen mmoles produced.

Vo: Estimated hydrogen mmoles produced by original inoculation.

Tk: Hydrogen production rate.

$\mathbf{T}(\mathbf{t}): \quad$ Time

Gw: Glucose consumed.

Gbc: Glucose consumed by first inoculation.

Cr: Consumption rate

QS: Quorum sensing (a cell to cell communication interaction).

\subsection{Equations Description}

The equations obtained are equations of difference for dynamic systems working on discrete periods of time, the term of time in the equations might be replaced by intervals of time that tend to the minimum unit of time used for the equation design e.g. In the equation 1 the time variable might be replaced for an interval of time that tends to 1 second if possible.

\subsection{Equations as Predictive Tools}

The equations shall be used as predictive tools as long as the system (bacterial culture) is maintained in the exponential phase of growth with mechanisms as hollow fiber cell culture [4]. This mechanisms act as a cell filter, which allows new sterile media to enter the system and the extraction of degraded media in order to purify, treat or eliminate it.

\subsection{Model Restrictions and Specifications}

The model is restricted as it does not consider all the possible factors that might change, interrupt, or stop the process of production, the restrictions of the model are as follows:

$>$ Pressure of the system.

> QS and other cell to cell communication or signaling interactions.

$>\quad$ Metabolites reaction that might interfere in the production process.

$>\mathrm{pH}$.

The following factors were not included in the model as they are going to be controlled in the experimental phase of this project (please refer to future remarks and perspectives section or contact the authors for more information).

$>$ Pressure of the system.

$>$ Temperature of the system.

$>\quad$ Hydrogen extraction.

$>\quad$ Glucose addition.

$>\quad$ Complete anaerobic growth conditions.

The model was designed for reactors that are capable of maintaining the exponential phase of growth of the bacterial culture.

\section{RESULTS}

\subsection{Growth Curve}

After the previously mentioned analysis of information using the data of growth kinetics from [1] 


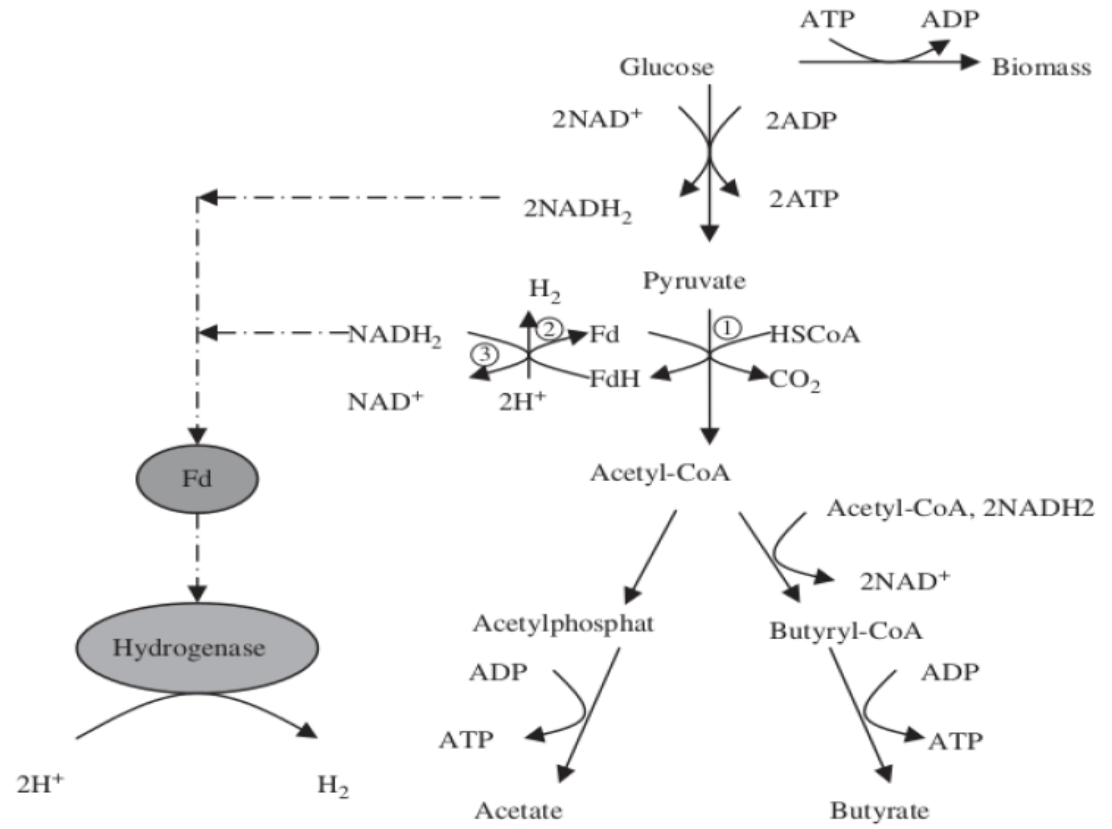

Figure 2: Metabolic pathways of hydrogen production in Clostridium butyricum [1].

the equation 1 (Eq. 1) was formed in order to adjust the data points to a tendency line (Figure $\mathbf{3 A}$ ), the $X$ values correspond to the time of interest in minutes, several other equations for using other units of time were also calculated and are presented in equations 1 and $1 \mathrm{~b}$ and, the equation presented in 1 has a $R^{2}=0.9783$, the $R^{2}$ values for the subsequent equations were not calculated as they do not form part of the objectives.

$P=N \cdot(T c)^{t}($ Population Eq.1)

$\rightarrow$ E.g. for time(hours).

$$
\begin{gathered}
f(x)=71421.0015 \cdot 2.8423^{x} \text { Eq.1a } \\
(x=\text { time }(\text { hours }))
\end{gathered}
$$

\subsection{Hydrogen Production Curve}

The curve of hydrogen (Figure 3B) production was obtained from the comparison between the stoichiometric analysis of production according to glucose consumption and the values coming from experimentation of other authors (data not shown) the selected data series was the one from [1] the data was standardized and a tendency line was generated, the information gathered was used in function of glucose consumed in the system -value regulated by the cell density and so by the time- in order to get a curve controlled by a previously controlled variable.

The equation for the previously mentioned curve is presented in Eq. 2, the rate of production is considered.
Equations might be modified to convenience in order to be adjusted to other units of time -or- other units of cell density.

$V=V_{o} \cdot\left(T_{k}\right)^{t}(E q \cdot 2)$

$\rightarrow$ E.g. for time(Minutes.).

$$
\begin{gathered}
f(x)=0,04317 \cdot 2 \cdot 2954^{x} E q \cdot 2 \mathrm{a} \\
(x=\operatorname{time}(\text { hours }))
\end{gathered}
$$

\subsection{Glucose Consumption}

A power regression (Figure $\mathbf{3 C}$ ) was used in order to determine the tendency curve of the glucose consumption process, an $\mathrm{R}^{2}=0.981$ was obtained in this curve, the equation is presented in Eq. 3 .

$$
\begin{aligned}
& G_{w}=G_{b c} \cdot P^{C r}(E q \cdot 3) \\
& \rightarrow \text { E.g. for Number of cells }(\text { CFU's }) . \\
& f(x)=0,00052 \cdot P^{0,5049} E q \cdot 3 \mathrm{a} \\
& \quad\left(x=\text { Cells }\left(C F U^{\prime} s\right)\right)
\end{aligned}
$$

\subsection{Carbon Dioxide, Acetate and Butyrate Curves}

The values of carbon dioxide, acetate and butyrate production were calculated using the following stoichiometric proportions [1]: 

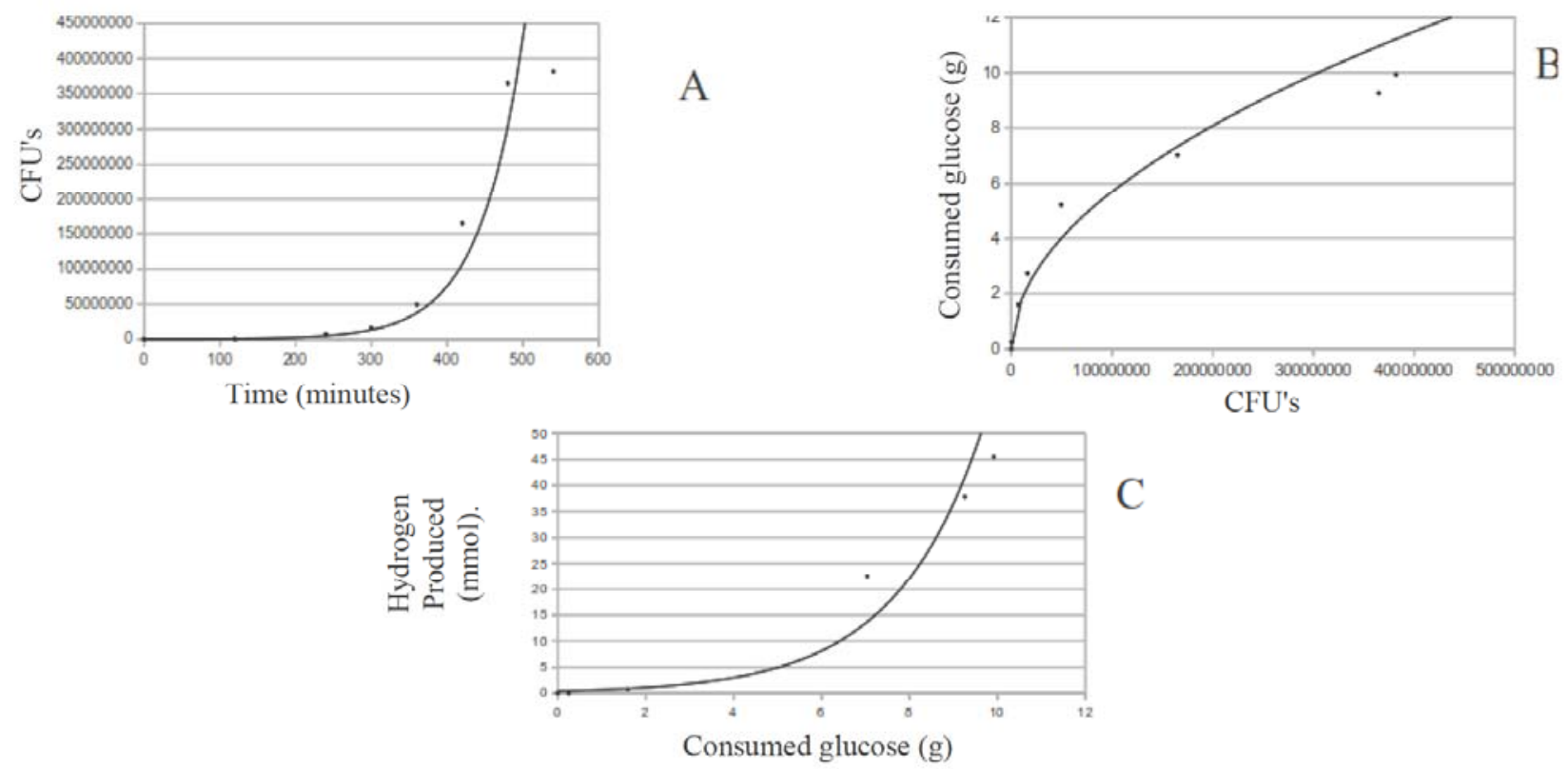

CFU's

Figure 3: Graphics for tendency lines based on experimental results from [1] A: Growth curve, B: Glucose consumption curve; C: Hydrogen production curve.

Acetate:

$$
\begin{gathered}
\mathrm{C}_{6} \mathrm{H}_{12} \mathrm{O}_{6}+2 \mathrm{H}_{2} \mathrm{O} \rightarrow 2 \mathrm{C}_{2} \mathrm{H}_{4} \mathrm{O}_{2}+2 \mathrm{CO}_{2}+2 \mathrm{H}_{2} \\
+4 \mathrm{ATP}+2 \mathrm{NADH}_{2} .
\end{gathered}
$$

Butyrate

$\mathrm{C}_{6} \mathrm{H}_{12} \mathrm{O}_{6} \rightarrow \mathrm{C}_{4} \mathrm{H}_{8} \mathrm{O}_{2}+2 \mathrm{CO}_{2}+2 \mathrm{H}_{2}+3 \mathrm{ATP}$.

This proportions generate a new one for carbon dioxide in which the proportion 1:1 generating for carbon dioxide production a curve almost identical to the one of hydrogen production.

As the curves for acetate and carbon dioxide are almost identical to the one for hydrogen production, the equations used are the same for hydrogen production (see Eq. 1).

\subsection{Curve Results Comparison}

As the results of the curves were obtained, data was stored and stacked in tables for posterior comparison within: curves of different units and curves generated for each one of the variables in simulated and experimental time.

In the case of hydrogen production the curve generated in relation of the kind of time considered (simulated or real) is presented in Figure $\mathbf{5}$. As it can be seen the curves adjust to each other in a tight relation $\left(R^{2}>0,97\right)$ when predicted results were obtained (data not shown) the values were still adjusted to the curve demonstrating that the curves may be useful for obtain of predictive results in function of time, initial culture inoculation, glucose present in culture media and other variables that might be connected to the equations here presented.

The curve in function of time presented in Figure $\mathbf{5}$ is product of overlapping the adjusted values of hydrogen produced in function of time in Figure $\mathbf{3}$ with the results obtained in the model by evaluation of discrete periods of time (near to 1 minute) in the equations designed.

All the results obtained in simulated time were stacked in tables and compared in order to expose the final results of the model evaluation until the minute 540 . Discrete periods of time tending to 1 minute were evaluated in the equations designed and all data was stored in tables (data not shown 540 calculations performed) data for curves was stored randomly and stacked in a different database for formation of curves.

The comparing curves are shown in Figure 6, if comparison is wanted, results in figure 6 might be compared to the ones in Figures $\mathbf{3 A}, \mathbf{3 B}$ or $\mathbf{3 C}$ as this results are product of standardization of experimental data and provide a reference point for curves discrimination, in all of the cases, as mentioned before $\mathrm{R}^{2}$ values of adjustment is always discriminated at $\sim 0,97$. 


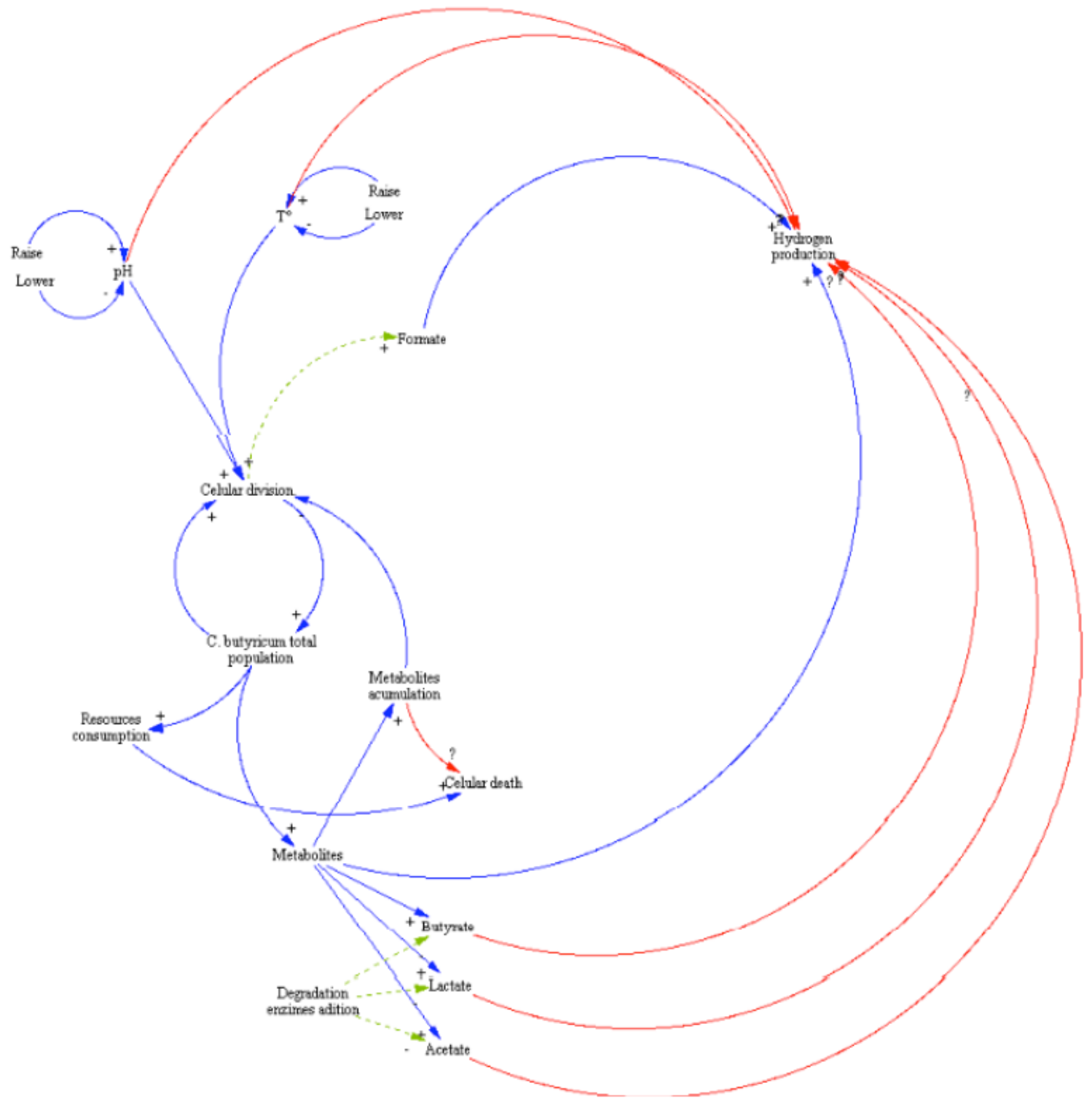

Figure 4: Relations diagram between the components of the hydrogen production system of Clostridium butyricum, positive arrows indicate a positive (raise) influence over the variable, negative ones indicate negative influences, dashed (green) ones indicate possible places to add degradation enzymes, lighter ones (red) indicate unknown relations of the variables, darker (blue) lines express known positive or negative relations between variables.

\section{DISCUSSION AND CONCLUSIONS}

The results obtained demonstrate that the modelation of $C$. butyricum hydrogen production process is useful in the optimization of the industrial production due to the possibility to analyze how each part of the system works and articulates with the other parts, in addition to that it's possible to maintain the exponential phase of the growth curve by using methods such as cell flows and substrate flows in the laboratory system or designing a new system for the industrial process, in addition to that is important to remark that the found behaviors of the curves are really close to the reality so that demonstrates that it is not necessary to implement complex optimizations to the process, the optimization should actually be focused over the treatment of metabolites such as acetate and butyrate with enzymes or bacterial consortiums so the hydrogen production can be taken to the upper limit, even if the performance of $C$. butyricum in hydrogen production is high compared to the production of other metabolites it's still necessary an optimization if the objective is to massively produce the metabolite of interest.

As the demands of energy sources (in diversity and quantity) are each day higher a first suggested method for optimization of hydrogen production is the 


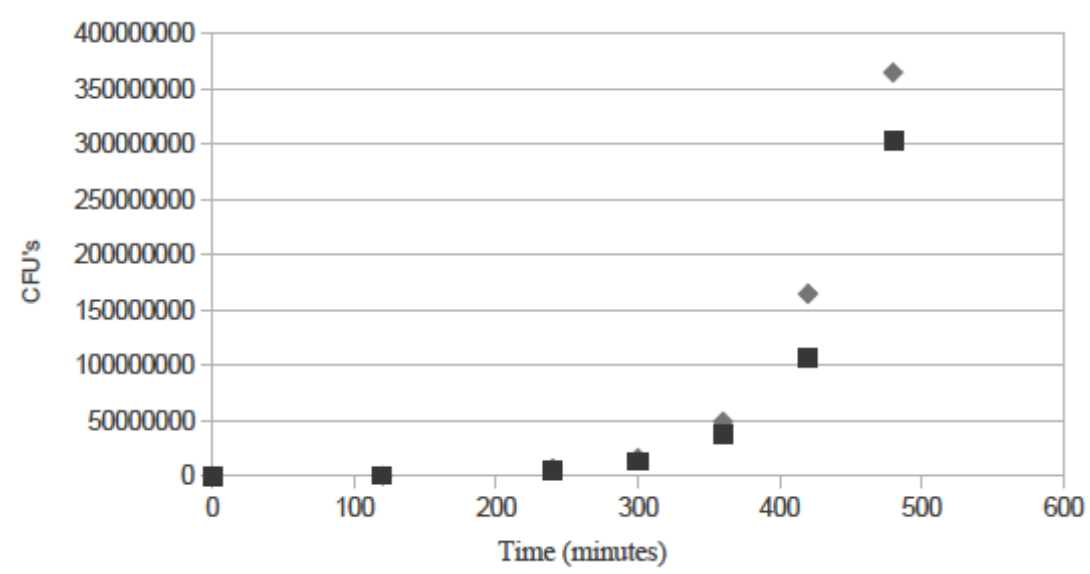

Figure 5: Poblation curve result of the evaluation of $X$ with values from the simulation compared to the one with experimental results $A$ : ideal curve; B: experimental adjusted curve. ( $\diamond$ : simulated time, $\square$ : experimental time).

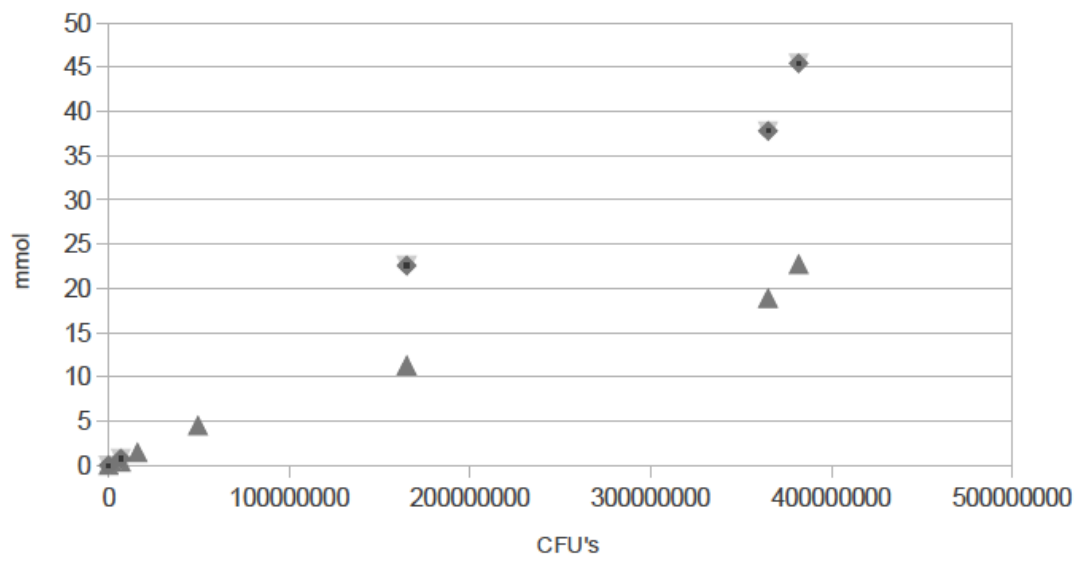

Figure 6: (Combined) Curves for hydrogen, acetate, butyrate and carbon dioxide production related with $C$. butyricum cell density. $(\square$ : Hydrogen, $\diamond$ : carbon dioxide, $\nabla$ : acetate, $\triangle$ : butyrate.

increment of available base resources for the bacterial culture and the look up for a way to improve the stoichiometric proportions of glucose consumption.

The previously reported results suggest that the process efficiency is quite high so the production with this bacterial species (Clostridium butyricum W5) is indeed possible, profitable, optimizable and implementable, the previous modelation work is the first of a series of steps that should be taken in the research of new energy sources production (as hydrogen) mediated by biological agents, as the prediction of the behavior of a system helps for the future implementation of it. To our knowledge this modeling process has not been made focusing in the future uses that it might have so the next step in our work is an experimental phase in which we will compare the real behavior of the system with the modeled one, after this comparison we will be able to detect factors that might have influence in the production and we will be able to start the research about this factors.

Some of those factors that we are starting to consider are: Cell to cell communication interactions, maximum population capacity (MPC) of the reactors or culture containers and $\mathrm{pH}$ and Temperature modifications.

It's strongly remarkable that optimum conditions of $\mathrm{pH}$ and temperature should be maintained in order to acquire the best hydrogen production performances and rates and of course in order to maintain the best performance in the growth curve.

As previously mentioned the use of $C$. butyricum, Kleibsella pneumoniae or Citrobacter freundii as a potential industrial or high scale hydrogen productors 
$[5,6]$ is possible and recommended because of the wide range of types of substrate that are consumable for it and because of the facility to be found in a wide range of environments.

Finally, the use of this species of bacteria in order to produce hydrogen from substrates of high glucose concentrations is promising in the social field because as it is mentioned previously it might be a good source of income for low income people all around the world (specially in tropical parts where the environmental conditions are easier to maintain and so are the culture conditions).

Agricultural residues might be used as culture media or substrates in order to produce hydrogen via C. butyricum W5 metabolism, this will be discussed in future phases of our project as the evaluation of the most optimum substrate are still pending, briefly the production process should be done in an anaerobic environment reachable by exposing air containers to chemicals that help in the reduction of the partial concentration of oxygen, such as sodium thioglycolate or L-ascorbic acid. After that hydrogen and other metabolites quantities might be measured by several methods.

In the experimental part of this project we plan to extract and purify the hydrogen (present in diatomic form $\left(\mathrm{H}_{2}\right)$ Sol. Water: $\sim 0,00155 \mathrm{~g} / \mathrm{Kg} \quad \mathrm{H}_{2} \mathrm{O}$ ) with molecular membrane filters so maximum purification is accomplished.

As the production of hydrogen changes in each phase of the growth curve and the growth curve changes to a stationary phase even if the resources for the cells are enough to keep the exponential growth, it can be discussed the presence of QS (Quorum Sensing) circuits (genetic expression regulatory circuits) that might be regulating the production or growth process of the $C$. butyricum population and also other cell to cell communication interactions.

The results obtained are comparable to the production reported by other authors with $C$. butyricum WT, $C$. butyricum W5 and any other species which production is made via the metabolic way exposed in the Figure 2 [7-9] in addition to that we can say that the curves obtained are actually really close to reality, because the descriptive equations have a $R^{2}$ value close to 1.00 which suggests us that if the predicted conditions are recreated we can actually get really good results in the experimental processes and also suggests us that with the modification of some of the variables including the substrate concentrations we can actually optimize the process.

\section{FUTURE REMARKS AND PERSPECTIVES}

For further studies it is strongly recommended to study and prove the presence or absence of QS genetic circuits or other cell to cell communication interactions gene circuits in the species used in this study in order to analyze the influence of them in the production process, in addition to that we strongly suggest to realize studies in order to maintain the variables that influence the hydrogen production process under control.

It's also strongly recommended that further studies focus in the modeling of other metabolic pathways in order to start a comprehensive study of all the possible ways of optimization.

After the development of this study an experimental one is going to be made in order to test the results obtained in the model, in addition to that a work about hydrogen production and storage is being planned.

The experimental phase of our project is briefly discussed in the discussion and conclusions section of this paper.

\section{ACKNOWLEDGEMENTS}

We would like to thank the developers of JMCAD for developing the modelation software and allowing it to be open-source, to the Universidad Nacional de Colombia for bringing us access to the necessary information databases, to our colleagues, specially to Diana P. Sanabria. Finally to the research group APRENA and the department of Biology of the Universidad Nacional de Colombia for the spaces to develop this work.

\section{INTEREST CONFLICTS}

None declared.

\section{ETHICAL APPROVAL}

Not required.

\section{REFERENCES}

[1] Chen X, Sun Y, Xiu ZL, Li X, Zhang D. Stoichiometric analysis of biological hydrogen production by fermentative bacteria. Int J Hydrogen Energy 2006; 31: 539-49. http://dx.doi.org/10.1016/j.ijhydene.2005.03.013 
[2] Wang X, Monis PT, Sainta CP, Jina B. Biochemical kinetics of fermentative hydrogen production by Clostridium butyricum W5. Int J Hydrogen Energy 2009; 34: 791-98. http://dx.doi.org/10.1016/j.jijhydene.2008.11.023

[3] Robinson PR, Dolbear GE. Hydrotreating and hydrocracking: fundamentals. In: Chang SH, Paul RR, Eds. Practical advances in petroleum processing 2006; pp. 177-218.

[4] Hopkinson J. Hollow Fiber Cell Culture Systems for Economical Cell-Product Manufacturing. Nat Biotechnol 1985; 3: 255-30.

http://dx.doi.org/10.1038/nbt0385-225

[5] Liu F, Fang B. Optimization of bio-hydrogen production from biodiesel wastes by Klebsiella pneumoniae. Biotechnol $\mathrm{J}$ 2007; 2: 374-80

http://dx.doi.org/10.1002/biot.200600102

[6] Thompson LJ, Gray VM, Kalala B, Lindsay D, Reynolds K, von Holy K. Biohydrogen production by Enterobacter cloacae and Citrobacter freundii in carrier induced granules. Biotechnol Lett 2008; 30(2): 271-74. http://dx.doi.org/10.1007/s10529-007-9527-y
[7] van Andel JG, Zoutberg GR, Crabbendam PM, Breure AM. Glucose fermentation by Clostridium butyricum grown under a self generated gas atmosphere in chemostat culture. Appl Microbiol Biotechnol 1985; 23: 21-26.

http://dx.doi.org/10.1007/BF02660113

[8] Pattra S, Sangyoka S, Boonmee M, Reungsang A. Biohydrogen production from the fermentation of sugarcane bagasse hydrolysate by Clostridium butyricum. Int $\mathrm{J}$ Hydrogen Energy 2008; 33(19): 5256-65.

http://dx.doi.org/10.1016/j.ijhydene.2008.05.008

[9] Karube I, Matsunaga T, Tsuru S, Suzuki S. Continuous hydrogen production by immobilized whole cells of Clostridium butyricum. Biochim Biophys Acta (BBA) 1976; 444(2): 338-43.

http://dx.doi.org/10.1016/0304-4165(76)90376-7 